\title{
Alinement of \\ Solid Interstellar Particles \\ by a Magnetic Field ${ }^{1}$
}

LYMAN SPITZER, JR.

Princeton University

Princeton, New Jersey

AND

R. V. Jones

Harvard University

Cambridge, Massachusetts

TOR a LONG Time the Davis-Greenstein theory (ref. 1) has been Fenerally considered an adequate explanation of the mechanism responsible for the alinement of interstellar grains and thus for the observed interstellar polarization. The magnetic field required to orient paramagnetic grains is apparently somewhat greater than $10^{-5}$ gauss, a relatively large field, but according to reference 2 ferromagnetic grains, proposed by the authors of reference 3 , can be oriented by a field of only $10^{-7}$ gauss. These results indicate that orientation of interstellar grains is easily explained, even if the magnetic field in interstellar space is relatively weak.

Unfortunately, this relatively satisfactory state of affairs has been upset by two developments. Firstly, the basic mechanism of magnetic relaxation proposed by Davis and Greenstein has been questioned by Dr. C. Kittel of the University of California, who has pointed out that the disorienting effect associated with thermal fluctuations of magnetization within the solid particles is ignored in the formulation of this mechanism. In thermodynamic equilibrium this effect cancels out the effect of the average magnetic torques in alining the grains, and the distribution is isotropic. Secondly, further analysis of ferromagnetic relaxation (ref. 4) has led to numerical corrections in the results of

1 The contents of this paper were published previously in the Astrophys. J. (published by the Univ. of Chicago Press); vol. 147, 1967, p. 943.

$271-9920-67-6$ 
reference 2, and it now appears that if magnetic relaxation can aline particles at all, a field of $10^{-5}$ gauss will be required for ferromagnetic particles, a value not much less than that required for paramagnetic grains. The present paper explores these two difficulties, analyzing the fundamental basis for magnetic alinement and reexamining the strength of the field required with ferromagnetic grains.

A detailed discussion of grain orientation, in which thermal fluctuations of magnetization as well as steady torques are considered, is beyond the scope of the present paper. Suffice it to point out here that grain orientation can be understood in terms of a steady-state distribution in which the rotational temperature that characterizes the distribution of rotational energy tends to be different for different directions of the rotational momentum $\mathbf{J}$. For $\mathbf{J}$ parallel to the magnetic field $\mathbf{B}$, there is no magnetic torque and the temperature is about equal to the gas temperature $T_{g}$; for $\mathbf{J}$ transverse to $\mathbf{B}$, a substantial magnetic torque can arise, and the kinetic temperature is somewhere between the gas temperature $T_{g}$ and the internal temperature $T_{i}$ of the grains. If these two temperatures are equal, there is no orientation of the rotational momentum, but if $T_{i}$ is much less than $T_{g}$, the grains will spin primarily about axes parallel to $\mathbf{B}$ if collisions with gas atoms are sufficiently infrequent.

Orientation of the principal axes of the grains relative to the angular momentum direction also follows from simple equilibrium consideration. If prolate spheroids are considered, the condition that the kinetic energy of rotation about the principal axis equals that about each of the transverse axes leads directly to the result that the angular momentum is systematically greater about the transverse axes, with their greater moment of inertia. The net result is that for prolate spheroids in equilibrium the principal axes tend to be perpendicular to the angular momentum vectors of each grain. The combination of this tendency with the alinement of $\mathbf{J}$ parallel to $\mathbf{B}$ produces the grain alinement predicted by the Davis-Greenstein theory.

A more quantitative discussion of paramagnetic relaxation, based on an idealized model, gives an equation for the orientation parameter $F$ introduced by Davis and Greenstein. The value of this parameter is $1 / 3$ for complete orientation and it vanishes for an isotropic distribution. For weak orientation $(F$ much smaller than $1 / 3)$ this equation becomes

$$
F=\frac{\chi^{\prime \prime} B^{2}}{75 a \omega n}\left(\frac{2 \pi}{m k T_{g}^{\prime}}\right)^{1 / 2}(\gamma-1)\left(\frac{T_{i}}{T_{g}}-1\right)
$$

where

$\chi$ " imaginary part of magnetic susceptibility

$a$ grain radius

$n$ number density of $\mathrm{H}$ atoms 
$m$ mass of $\mathrm{H}$ atom

$k$ Boltzmann constant

Except for a relatively minor change in numerical constants, equation (1) agrees with equation (90) of reference 1 except for the factor $\left(T_{i} / T_{g}\right)-1$. The value of the present theory is that it provides a sounder physical basis for this result and indicates how the alinement depends on the difference between $T_{i}$ and $T_{g}$. It is assumed that similar results apply also for ferromagnetic and other anisotropic grains, although the present analysis has not been applied specifically to such particles.

A reexamination of ferromagnetic relaxation rates has led to a redetermination of the magnetic susceptibility, particularly its imaginary part $\chi^{\prime \prime}$, which is responsible for a dissipation of energy when a particle rotates in a magnetic field. Theoretical and experimental research on this subject has much increased our knowledge since the original work of reference 2 . The dissipation of energy results from scattering of energy out of a uniform rotation mode into other normal modes of the particle, particularly the lattice vibrations and spin fluctuations. A study of these scattering processes indicates that the value of $\chi^{\prime \prime}$ adopted in references 2 and 4 was probably too small by several orders of magnitude. In view of the uncertainty as to the structure of interstellar grains, definite numerical values cannot be cited, but it appears quite likely that substantial orientation of interstellar grains can be achieved in magnetic fields of $10^{-6}$ gauss or less.

\section{REFERENCES}

1. Davis, L.: and Greenstein, J.: Polarization of Starlight by Aligned Dust Grains. Astrophys. J., vol. 114, 1951, p. 206.

2. Henry, J.: Polarization of Starlight by Ferromagnetic Particles. Astrophys. J., vol. 128,1958, p. 497.

3. SPITZER, L.; and TUkey, J.: A Theory of Interstellar Polarization. Astrophys J., vol. 114, 1951, p. 187.

4. Cugnon, P.: Sur la Polarisation de la lumière des étoiles. Bull. Soc. Royale d. Sciences Liege, vol. 32, 1963, p. 228.

\section{DISCUSSION}

Field: Did you say that the effect of the thermal fluctuations would be small?

Spitzer: The effect of the thermal fluctuations is to cancel the orientation completely if the grain temperature is equal to the gas temperature. If these two temperatures are equal, the grains act as though they are in a thermodynamic enclosure as far as all rotational motions are concerned and there can be no orientation. The effect of thermal fluctuations is small, as assumed by Davis and Greenstein, if the grain temperature is much smaller than the gas temperature. As a first approximation, the orientation is determined by the difference between the grain temperature and the gas temperature. 
Greenberg: If the grain temperature is $10^{\circ}$ and the gas temperature is $100^{\circ}$, the effect is negligible. If, on the other hand, the grain temperature is comparable to the gas temperature, then one should get no polarization. This might be one of the distinguishing features of the various types of grains, because various models of grains adopt quite different grain temperatures.

There are two effects: magnetic susceptibility is itself temperature sensitive and this temperature sensitivity is different depending on whether the grains are paramagnetic or diamagnetic. Also, different kinds of grains have different grain temperatures relative to the temperature of the gas. Therefore, the dependence of polarization on the ambient physical (radiation) conditions would be different for various grain models.

Donn: If we did have some relatively hot cloud that was warmed up by some mechanism where the gas temperature got high but the grains were not destroyed, then this would be a mechanism whereby one may have a high or low polarization depending on the temperature of the cloud.

Spitzer: If the internal grain temperature exceeds the gas temperature, then the polarization is in the opposite direction.

Greenberg: Is this really true or is it only a first-order approximation?

Spitzer: I should emphasize, perhaps, that the model I have presented is really not exact, since a full solution of the Fokker-Plauck equation has not yet been possible, but it does at least give results for arbitrary values of the temperatures and other parameters involved. The model predicts a linear variation of alinement only when the alinement is weak.

Wickramasinghe: Do you expect the same analysis to apply to the graphite particles?

Spitzer: The analysis in its present form should be applicable to any isotropic particles, but not to anisotropic particles such as graphite. When the grain temperature is equal to the gas temperature, orientation must disappear for all types of particles. It should be possible to work out an approximate theory for graphite.

Elvius: Would these numbers for the magnetic field apply to paramagnetic particles?

Spitzer: For paramagnetic particles, one gets the same magnetic field strength that Davis and Greenstein originally found, which is an embarrassingly high $10^{-5}$ gauss. However, some believe that the interstellar magnetic field probably has this high value.

Greenberg: I once calculated the fields needed to orient the graphite type of particles and I did not find that the fields that were required significantly less than those for dirty ice particles. 